\title{
Kernos
}

Revue internationale et pluridisciplinaire de religion grecque antique

$10 \mid 1997$

Varia

\section{Le mythe de Phaéthon}

\section{Jean Rudhardt}

\section{OpenEdition}

\section{Journals}

Édition électronique

URL : http://journals.openedition.org/kernos/649

DOI : $10.4000 /$ kernos.649

ISSN : 2034-7871

Éditeur

Centre international d'étude de la religion grecque antique

Édition imprimée

Date de publication : 1 janvier 1997

Pagination : 83-95

ISSN : 0776-3824

Référence électronique

Jean Rudhardt, « Le mythe de Phaéthon », Kernos [En ligne], 10 | 1997, mis en ligne le 12 avril 2011,

consulté le 30 avril 2019. URL : http://journals.openedition.org/kernos/649 ; DOI : 10.4000/kernos.649

Kernos 


\section{Le mythe de Phaéthon}

On connait l'aventure de Phaéthon, telle qu'Ovide la raconte ${ }^{1}$. Fils du DieuSoleil mais né d'une femme, il est mortel. Or il emprunte le char de son père. Incapable d'en maitriser les chevaux, il se laisse emporter hors de la trajectoire que l'astre doit suivre. Tour à tour trop près des constellations ou trop près des terres, il menace d'embraser le monde. Jupiter le tue d'un coup de foudre. Emprunté à la Grèce où il est traditionnel, relatant des événements vécus par des dieux ou des personnages doués de pouvoirs surhumains, ce récit est ce que l'on est convenu d'appeler un mythe.

De nombreux auteurs ont mis en évidence l'universalité des mythes. Non seulement, notent-ils, presque toutes les cultures en ont possédé mais de mêmes motifs mythiques se retrouvent chez des peuples que nulle relation semble n'avoir jamais unis dans le cours de l'histoire. Un maître de la mythologie comparée, James Frazer, a notamment rapproché le mythe de Phaéthon de récits collectés à la fin du siècle dernier par Franz Boas, un des pères de l'anthropologie moderne, chez des Indiens de la côte nord-ouest de l'Amérique du nord ${ }^{2}$. Voici le plus remarquable d'entre eux.

Mariée contre son gré, une jeune femme épousa un personnage nommé Souche. Leur entente ne dura pas. La chevelure de Souche était pleine de gros champignons et il attendait de sa femme qu'elle les choisisse pour lui. C'était plus qu'elle ne pouvait supporter. Elle s'enfuit, poursuivie par Souche, trop confiant. Il gagnait du terrain mais elle sut retarder sa poursuite, en jetant successivement par-dessus son épaule une vessie pleine de liquide, un peigne et une pierre de meule. Le liquide forma un lac; le peigne donna naissance à un fourré; la pierre devint une grande montagne qui souleva la femme et lui fit atteindre le ciel. Là, elle parvint à la maison du Soleil; en guignant à travers une fente, elle vit le Soleil assis à l'intérieur, ressemblant à un homme. Il dit: «Entre! », mais, comme la porte était en flamme, elle hésita. Le Soleil lui dit de sauter à travers le feu; elle le fit et entra dans la maison, saine et sauve. Souche arriva après elle et, comme il tentait de franchir la porte incandescente, il fut consumé par les flammes. Alors la femme vécut dans un coin de la maison du Soleil et, au bout d'un certain temps, elle donna naissance à un garçon, le fils du

OVIDE, Métam., I, 747-752; 332.

2 J.G. Frazer (éd.), Apollodore. The Library, t. II p. 388-394: Appendix XI : Phaethon and the Stu. 
Soleil. Son nom était Totqoaya. Il était très laid et son visage était couvert d'ulcères.

Le moment vint où la femme désira retourner chez son père sur la terre. Selon les instructions du Soleil, elle prit son fils sur son dos et elle descendit le long des cils du Soleil qui sont les rayons de lumière, jusqu'à ce qu'elle arrive le soir à la maison de son père. Ses parents et ses amis furent très heureux de la voir.

Le lendemain matin, le garçon sortit de la maison pour jouer avec les autres enfants; or ils le tournèrent en ridicule. Alors il leur dit que son père était le Soleil mais ils se moquèrent de lui, au point qu'il en fut très fâché. Ensuite il dit à sa mère qu'il avait l'intention de retourner chez son père, dans le ciel. Il se fabriqua de nombreuses flèches et un arc, sortit de la maison et se mit à tirer des flèches vers le haut. La première se ficha dans le ciel; la deuxième se ficha dans l'encoche de la première; il continua de tirer jusqu'à ce qu'une chaîne de flèches soit formée et qu'elle touche le sol. Alors il grimpa puis, après avoir atteint le ciel, il se rendit dans la maison de son père. Là, il dit : "Père, je veux te remplacer demain! » Le Soleil y consentit mais il dit : «Prends soin de ne pas brûler les gens. J'utilise une seule torche le matin, puis j'augmente le nombre des torches jusqu'à midi. Pendant l'après-midi, je les éteins une à une ». Le lendemain matin, le garçon prit les torches de son père et se mit en marche sur le chemin du soleil; or il alluma très vite toutes les torches. Cela devint très chaud sur la terre. Les forêts commencèrent à brûler, les rochers, à craquer, et de nombreuses personnes moururent. Cependant sa mère agita les mains et garda de cette façon la fraîcheur de sa maison; les gens qui étaient entrés dans sa maison furent sauvés. Quand le Soleil vit ce que le garçon faisait, il le saisit, le jeta vers la terre et dit. « À partir de maintenant, tu seras le vison $»^{3}$.

Frazer a certainement raison de rapprocher le mythe indien du mythe raconté par Ovide. Il est intéressant de constater que deux cultures, éloignées l'une de l'autre dans l'espace et dans le temps, inventent également l'histoire d'un fils du Soleil qui, jouant le rôle de son père, le remplit mal et incendie le monde. Les deux mythes nous donnent à penser que les hommes reconnaissent l'importance du soleil, dispensateur de chaleur et de lumière; qu'ils l'admirent et le redoutent. Indispensable à la vie, il pourrait aussi la détruire; il doit conserver une juste mesure pour être salutaire. On devine la présence nécessaire d'un être fort, capable de sagesse et de maîtrise de soi, pour assurer le respect de cette juste mesure. Les deux récits illustrent aussi le désir qu'un fils peut éprouver d'imiter son père, voire de s'assimiler à lui; ils mettent du même coup en évidence l'impossibilité d'une telle assimilation. La comparaison du récit fait par Ovide et de celui qu'un ethnologue a recueilli chez des Indiens fait donc apparaitre deux de leurs significations latentes; l'une cosmique, l'autre psycho-

3 Dans une version légèrement différente du même récit, c'est le Soleil lui-même qui dit à son fils : "Je suis vieux. Désormais porte le Solell à ma place! Mais prends soin de..., etc. » 
logique. Quant au reste les deux mythes présentent des divergences notables et chacun d'eux requiert une exégèse particulière. Pour devenir intelligible, il doit être situé dans le contexte d'une culture dont il est solidaire. Que signifie le nom Souche, porté par le premier mari de celle qui deviendra l'épouse du Soleil ? Que sont les champignons qui poussent sur son corps? Qu'est-ce que ce jeune homme qui doit devenir un vison ? Pour le comprendre nous devrions étudier la fonction de la souche, celle des champignons, celle du vison dans la société, peut-être totémique, où le mythe est raconté. De même, l'attelage du récit classique se comprend dans une civilisation où l'usage du char attelé, char de guerre ou de parade, est le privilège d'une classe dirigeante; il y a quelque chose de seigneurial dans le Dieu-Soleil hellénique. Nous devrons en outre situer chaque mythe dans l'ensemble des récits qui habitaient la pensée de ceux qui les ont écoutés et transmis. Comme nous l'avons dit, Ovide emprunte le mythe de Phaéthon à la Grèce; il a rempli sa fonction originelle à l'intérieur d'un ensemble ordonné de mythes grecs. C'est ce que nous considérerons en premier lieu.

\section{Phaéthon dans les textes grecs}

Participe d'un verbe inusité à des formes conjuguées, le mot $\varphi \alpha \varepsilon ́ \theta \omega v$ veut dire lumineux, resplendissant. Dans l'Iliade, employé comme un adjectif, ce participe qualifie le Dieu-Soleil, Hélios ${ }^{4}$. Dans l'Odyssée, substantifiées, la forme

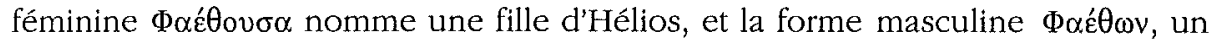
cheval de la Déesse-Aurore, Éos ${ }^{5}$. En bref, le héros Phaéthon est inconnu des poèmes homériques mais son nom s'y associe à ceux du Soleil et de l'Aurore.

Ce héros apparaît pour la première fois chez Hésiode, à la fin du viI ${ }^{\mathrm{e}}$ siècle; nous le trouvons ensuite à toutes les époques chez plusieurs écrivains grecs; il est en outre représenté sur de nombreux monuments figurés.

Selon l'enseignement de la Théogonie hésiodique, Phaéthon est né de Céphalos et d'Éos, la Déesse-Aurore ${ }^{6}$. La tradition dominante à partir de l'époque classique ${ }^{7}$ voit en Phaéthon un fils d'Hélios, le Dieu-Soleil, et lui donne pour mère une certaine Clyméné.

Les deux généalogies ne sont pas aussi différentes qu'il le paraît et nous fournissent en définitive des indications convergentes. Considérons en effet de plus haut les lignées divines. Uni à sa sœur Théia, le Titan Hypérion engendre

4 HOMËRE, Il., XI, 735.

5 HOMÈRE, Od., XII, 131-136; XXIII, 246.

6 HÉSIODE, Théog., 986-987. Cette tradition présente deux variantes mineures : un mythe attique appelle Héméra, Déesse-jour et non Éos, Déesse-aurore, l'amoureuse qui donne Phaéthon pour fils à Céphalos (PAUSANIAS, I, 3, 1). Le mythographe APOLLODORE (III, 14, 3) présente en Phaéthon un petit-fils, non un fils, de Céphalos et d'Éos.

7 Elle est même attestée dans un fragment attribué à Hésiode. HÉsıoDE, fr. 387 MerkelbachWest. 
Hélios, le Dieu-Soleil, Séléné, la Déesse-Lune, puis Éos, la Déesse-Aurore ${ }^{8}$. Le Soleil et l'Aurore sont donc frère et sœur. Il en résulte que, selon les deux traditions concurrentes, Phaéthon est soit fils d'Éos et neveu d'Hélios, soit fils d'Hélios et neveu d'Éos. Dans les deux cas il est le petit-fils du Titan Hypérion un personnage dont le nom signifie soit qui est plus élevé, soit qui va audessus des choses, qui se meut dans la bauteur ${ }^{9}$.

Enfants des dieux primordiaux, Ciel et Terre, les Titans sont des êtres formidables mais ils restent inaccomplis. À la fois cosmiques et divins, ce sont des entités cosmiques mal définies, des figures divines mal dessinées, et l'on ne peut définir clairement les fonctions que chacun d'eux remplit. Peu différenciés, ils n'accomplissent pas d'exploits individuels, lors des grands conflits mythiques où ils agissent collectivement ${ }^{10}$. Après leur défaite, ils sont rejetés aux limites inférieures du monde; seuls présents dans les régions centrales de l'univers, leurs descendants y exercent aujourd'hui une action immédiate. Le rôle des Titans fut ainsi d'engendrer des dieux dont la nature s'apparente sans doute à la leur mais qui, plus nombreux et plus divers, sont chacun mieux spécifiés, plus efficaces. Nous discernons mal les traits du Titan Coios et ceux de sa sour Phoibé, dont le nom signifie pourtant la brillante; la figure de leur fille Léto est mieux dessinée; unie à Zeus elle donne le jour aux dieux puissants et lumineux, Apollon - que l'on appelle aussi Phoibos - et Artémis - invoquée parfois sous le nom de Phoibé ${ }^{11}$; comme eux, elle reçoit des cultes en plusieurs lieux de la Grèce. Nous ne pouvons rien dire du 'Titan Hypérion, si ce n'est qu'il occupe la hauteur ou se déplace au-dessus des choses; sa figure reste vague; comme lui, ses enfants, Soleil, Lune, Aurore, se meuvent au-dessus de nos têtes, mais ils sont mieux délimités que lui et clairement visibles. D'une génération à l'autre, les êtres qui paraissent dans le cours de la théogonie sont donc mieux spécifiés; ils sont aussi plus limités. Dieu des floraisons, dieu de l'équilibre et de la lumière mais aussi dieu des prophéties et des purifications, Apollon est en outre un dieu guérisseur. Son fils Asclépios excelle dans cette dernière fonction mais il n'a pas les autres pouvoirs de son père; ce n'est plus qu'un dieu-médecin. À la troisième génération après les Titans (ou à la quatrième, dans les cas extrêmes), leurs descendants, parfaitement spécifiés, limités dans l'espace et dans le temps, n'ont plus qu'une existence de fait : ils sont mortels. À cet égard, la deuxième génération

8 HÉsIODE, Théog., 371-374.

9 Le mot peut être compris comme un comparatif (cf. latin superior); L'Etymologicum Magnum le présente comme le participe d'un verbe formé avec le préfixe byper sur le radical d'eimi, aller.

10 Seuls Cronos et son épouse, Rhéa, qui constituent le couple royal remplissent une fonction distincte parmi les Titans; seuls, ils conservent aujourd'hui une petite présence cultuelle. Les figures d'Océanos et de Téthys sont relativement bien dessinées; ils conservent une présence cosmique, serait-elle lointaine; mais, si Hésiode les situe parmi les Titans, d'autres traditions importantes leur attribuent un autre rang. Ce ne sont pas de vrais Titans. Cf. J. RUDHARDT, Le thème de l'eat primordiale dans la mytbologie grecque, Berne, 1971.

OPPIEN, Cynég., II, 1. 
occupe une position ambiguë; nous y voyons apparaître tantôt des mortels, tantôt des immortels. Si les petits-enfants du Titan Cronos sont encore de grands dieux, ceux du Titan Japet sont des divinités mineures ou simplement des êtres humains ${ }^{12}$. A cette génération, la limite entre le mortel et l'immortel est mal tracée; ni leurs positions respectives ni les modalités de leurs relations réciproques ne sont encore définies.

Phaéthon appartient à cette génération ambiguë. Selon Hésiode, uni à Perséis, le Dieu-Soleil, Hélios, engendra une divinité mineure, Circé - la magicienne dont Ulysse déjouera les ruses ${ }^{13}$. Selon d'autres auteurs, uni à Clyméné, Hélios engendra Phaéthon qui est mortel. Notre héros se situe bien dans cette zone frontière où le mortel et l'immortel, le divin et l'humain, sont dangereusement proches l'un de l'autre.

Le comportement d'Éos, mère ou tante de Phaéthon, me paraît significatif à cet égard. Déesse, elle s'unit à des hommes et ses unions aboutissent à d'étranges résultats. Éprise du beau Tithonos, elle l'enlève et, pour le garder toujours auprès d'elle, elle demande à Zeus de l'immortaliser. Celui-ci accède à sa prière; Tithonos devient immortel mais il vieillit et vieillit sans cesse, dans le cours de son immortalité ${ }^{14}$. Entre le mortel et l'immortel, l'amour aboutit à une impasse.

' Phaéthon lui-même est étroitement lié au jeu de ces amours inégaux. Voici en effet ce que nous lisons dans la Théogonie :

Pour Céphalos, Éos mit au monde un fils resplendissant, le robuste Phaéthon, un homme semblable aux dieux.

Encore adolescent, paré de la fleur d'une glorieuse jeunesse,

et sujet à la timidité, Aphrodite amie des sourires

le ravit et l'emporta dans ses temples divins; elle fit de lui, gardien nocturne du sanctuaire, un esprit divin. ${ }^{15}$

Dénonçant l'immoralité des dieux du paganisme, un auteur chrétien, Clément d'Alexandrie soulignera l'inconduite d'Aphrodite qui eut des aventures

12 Descendants de Japet : $1^{\text {re }}$ génération : le dieu révolté, Prométhée $: 2^{\mathrm{e}}$ génération le mortel Deucalion. $1^{\text {re }}$ génération: Atlas; $2^{\mathrm{e}}$ génération : divinités féminines, les Pléiades; $3^{\mathrm{e}}$ génération, enfants des Pléiades: un dieu: Hermès; des mortels, tels que Lacédaemon, Iasion ou Dardanos. Cf. APOLLODORE, I, 2, 4; I, 7, 2; III, 10, 1; III, 10, 2; III, 12, 1.

13 HÉSIODE, Théog., 956-957; 1011-1013.

14 HOMÈre, Il., XI, 1 sq.; Od., V, 1 sq. Hymne bomérique à Apbrodite, 218 sq. (Je signale qu'après s'être unie à Tïthonos, Éos donne le jour à Memnon, un roi d'Éthiopie que nous retrouverons dans le mythe de Phaéthon). Éos a d'autres aventures; elle tombe amoureuse du mortel Cephalos et le ravit. Selon la Théogonie d'Hésiode, c'est de lui qu'elle conçoit Phaéthon, comme nous l'avons déjà dit, mais, trop inégale leur union ne peut durer; Céphalos abandonne bientôt la déesse, pour épouser une femme mortelle. Ainsi, dans l'entourage de Phaéthon, la relation entre le divin et l'humain n'a pas encore trouvé les modalités qui la rendront viable.

15 HÉsIODE, Théog., 986-991. 
avec Arès, avec Cinyras, avec Anchise et avec Phaéthon. À propos de ce dernier,

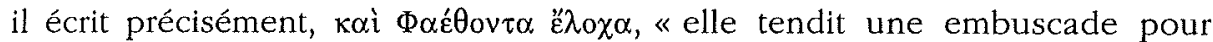
capturer Phaéthon. ${ }^{16}$ Nous comprenons mal le mythe auquel ces textes se réfèrent; il évoque une relation amoureuse difficile entre un mortel et une immortelle. Plus subtile qu'Éos, Aphrodite paraît trouver le moyen de garder auprès d'elle l'homme qu'elle a ravi; elle n'en fait pas un amant mais le reçoit dans son temple, écrit Hésiode, et lui confie une tâche quasi sacerdotale; du même coup, elle l'élève au-dessus de l'humanité commune.

$\mathrm{Au} \mathrm{V}^{\mathrm{e}}$ siècle, Euripide a consacré une tragédie à Phaéthon; elle a malheureu sement disparu mais nous en conservons plusieurs fragments ${ }^{17}$. Le poète fait de Phaéthon un enfant de Clyméné. Celle-ci, dit-il, a épousé Mérops, un roi des Éthiopiens, mais elle s'est aussi unie au Dieu-Soleil Hélios, dont Phaéthon est le fils. Le jeune homme l'ignore et tient Mérops pour son père. Après avoir refusé d'épouser Aphrodite, il y consent, quand Clyméné lui révèle sa véritable identité : il est fils d'Hélios. Clyméné lui conseille d'aller rendre visite à son père; elle prédit que le dieu, pour une raison que les fragments conservés ne nous font pas connaître, ne pourra pas refuser à son fils le privilège que celui-ci lui demandera. Phaéthon prie Hélios de lui confier son char. Le jeune homme monte dans l'attelage du Soleil et il entreprend de le conduire mais, incapable de le maîtriser, il dévie de sa route, finit par tomber sur le sol et meurt. Nous ne savons pas si c'est sous l'effet d'une intervention directe de Zeus.

Dans la pièce d'Euripide un nouveau thème s'ajoute donc à celui de l'union d'une déesse avec un mortel, le thème solaire que nous voyons développé par Ovide $^{18}$. Pour le comprendre, il faut encore une fois situer l'échec du cocher Phaéthon dans l'histoire de la génération médiane, où les rôles respectifs des hommes et des dieux ne sont pas clairement définis.

Il existe une parenté entre le grand père Hypérion, le fils Hélios et le petit-fils Phaéthon. L'usage de la langue le montre: dans les textes grecs, nom du grand-

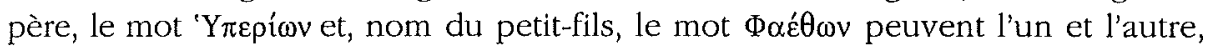
employés adjectivement, qualifier Hélios. En vertu de sa race, Phaéthon est naturellement enclin à revendiquer le privilège de conduire l'attelage solaire. Toutefois la délimitation qui de génération en génération contribue à spécifier les êtres dans le cours de la théogonie fait de lui un mortel. Il a certainement des affinités avec le ciel et la lumière mais cette noblesse et cette splendeur doivent elles se manifester dans le cosmos? ou peuvent-elles seulement le faire parmi les hommes? L'échec de sa tentative enseigne qu'en dépit de leur parenté initiale, les mêmes conduites ne conviennent pas aux hommes et aux dieux. Son aven-

\footnotetext{
16 Clément D'Alexandrie, Protr., II, 33, 9.

17 EuRIPIDE, Tragcediae, t. III, fr. 771 à 784 Nauck $^{2}$.

18 En Grèce même, repris par de nombreux auteurs, de Platon et d'Aristote à Diodore et à Quintus de Smyrne, il paraît plus populaire que celui du mariage divin.
} 
ture s'inscrit dans la série des événements mythiques propres à définir les comportements respectifs des mortels et des immortels.

Dans le mythe de Phaéthon, le motif du char du Soleil et celui des unions malheureuses entre une déesse et des hommes donnent donc des enseignements convergents. Dans la pièce d'Euripide, les deux thèmes s'articulent en outre avec logique. Phaéthon est mortel mais il est fils d'un dieu; cette parenté pourrait diminuer la distance qui le sépare d'Aphrodite. En effet quand le jeune homme apprend qu'il est d'ascendance divine, l'inquiétude que l'idée d'un mariage avec une déesse lui inspirait se dissipe; rien, pense-t-il, ne devrait s'y opposer. Tout semble donc conclu et les préparatifs de la noce vont bon train. Or, à ce moment, Clyméné envoie son fils auprès du Dieu-Soleil. Espère-t-elle que cette visite le confortera? Éprouve-t-elle inconsciemment un doute: Phaéthon possède-t-il les qualités de son père ? L'épreuve du char doit le révéler. Or elle met cruellement en évidence la faiblesse du jeune homme. Le mariage inégal n'aura pas lieu ${ }^{19}$.

Nous avons concentré notre attention sur Phaéthon; il convient que nous la portions maintenant sur le monde et sur les hommes.

Les mythes prométhéens racontent la séparation des dieux et des hommes puis l'établissement entre eux de rapports nouveaux. L'un d'entre eux est celui du déluge. Les Grecs connaissent plusieurs déluges, tous partiels; le plus important fut celui de Deucalion. Or Deucalion était un fils de Prométhée. Averti par son père de l'imminence du déluge, il construisit un vaste coffre où il embarqua avec sa femme et ses enfants, le moment venu. Comme Noé, il survécut ainsi à l'inondation. Ses enfants seront les ancêtres éponymes des grandes races helléniques. Les récits sémitiques du déluge, parmi lesquels il faut situer le récit biblique, ont certainement influencé le mythe grec mais celui-ci diffère du récit biblique sur un point essentiel. Selon la Genèse, Dieu fait le déluge pour anéantir une humanité corrompue et sauve en Noé le seul juste, pour rendre possible le développement d'une humanité meilleure. Le mythe grec ne prononce de jugement moral ni sur l'humanité ni sur la personne de Deucalion et ne justifie pas toujours l'inondation. Elle s'inscrit dans la série des événements mythiques qui établissent une juste distance entre les mortels et les immortels, qui définissent

19 Peut-être Aphrodite ne reste-t-elle pas sur cet échec. Dans la tragédie d'Euripide, le chœur lui adresse ces paroles : "Je te dois ces chants nuptiaux, ô Cypris, la plus belle des déesses, à toi et à ton époux, celui que tu caches dans l'éther». Nous ignorons à quel moment du drame, le chœur interpelle Aphrodite de cette façon. Pour les Grecs, l'éther est un air très pur, sec et lumineux, qui occupe les régions supérieures de l'univers. Nous voyons mal dans quelle circonstance, la déesse y a logé l'homme dont elle était amoureuse. Peut-être faut-il rapprocher ce fragment énigmatique des vers non moins énigmatiques d'Hésiode que j'al cités : «Aphrodite le ravit et l'emporta dans ses temples divins; elle fit de lui, gardien nocturne du sanctuaire, un esprit divin». Aphrodite est la déesse de la planète qui porte son nom et que nous appelons du nom latin correspondant, Vénus. Cette planète serait-elle le sanctuaire mentionné par le poète? Au-delà de la mort, la déesse associerait-elle à son astre le lumineux Phaéthon? L'hypothèse est séduisante mais elle reste incertaine à mes yeux. 
les modalités de leurs relations et contribuent à conférer une ordonnance aux sociétés humaines ${ }^{20}$.

Certains textes antiques nous donnent à penser que l'on a parfois attribué une signification analogue au mythe de Phaéthon. Selon Platon, les prêtres Égyptiens enseignèrent à Solon que les peuples ont subi plusieurs destructions successives, les unes sous l'action de l'eau, les autres sous l'action du feu ${ }^{21}$. Or, parmi ces dernières, ils ont mentionné l'incendie provoqué par la maladresse du fils d'Hélios ${ }^{22}$. Cet incendie pourrait donc bien avoir une fonction comparable à celle du déluge.

Les vieilles catastrophes mythiques ont laissé des traces dans le monde. Le déluge, par exemple a façonné le relief de la Thessalie. De même, l'incendie provoqué par Phaéthon a détruit de nombreux astres dont les restes constituent la voie lactée, selon des philosophes pythagoriciens ${ }^{23}$; la chaleur d'un soleil trop proche a brûlé le visage de plusieurs peuples dont la peau reste noire depuis $\operatorname{lors}^{24}$. Ces traces visibles rappellent aux hommes les événements qui ont défini leur condition. La chose n'est pas inutile car ces événements complexes auxquels Phaéthon fut mêlé demeurent actuels. Il n'ont pas seulement instauré une juste distance entre les hommes et les dieux; ils ont défini du même coup une sorte de mesure que les mortels doivent conserver dans toutes leurs conduites, aussi bien que dans leur croissance, une règle d'équilibre et de lucidité, proportionnant les desseins humains à la force du sujet qui les conçoit.

Parmi les vers conservés du Phaéthon d'Euripide nous trouvons une application de ce principe. «Folies chez les mortels : laisser une pleine liberté à des enfants ou à des citoyens qui ne sont pas raisonnables ».

20 Certaines traditions lient le déluge à l'impiété des fils de Lycaon; or les mythes de Lycaon, par l'image d'une rupture du lien d'hospitalité, symbolisent, comme ceux de Prométhée, la séparation des hommes et des dieux. Sur le déluge, voir l'ouvrage classique, même s'il est un peu vieilli : H. USENER, Sintfutsagen, Bonn, 1899. Sur la fonction de Deucalion, voir en outre J. RUDHARDT. Les mytbes grecs relatifs à l'instauration du sacrifice: les rôles corrélatifs de Prométhée et de son fils Deucalion, in $M H, 27$ (1970), p. 1-15.

21 Platon, Timée, 22c.

22 Parlant du conflit permanent qui, dans le monde, oppose l'eau et le feu, deux principes élémentaires dont les forces finalement s'équilibrent, Lucrèce se réfère au mythe du déluge et à celui de Phaéthon (LUCRÈCE, De Rerum Natura, V, 396-405) Hygin mentionne les deux mythes dans une même série. HYGIN, Fabulae, 152 et 153.

23 ARISTT., Météorol., A 8, 345a $13=41$ OINOPIDES 10 D.K.; cf. Pythagorische Scbule, 37c D.K.; voir aussi DIODORE DE SICILE, V, 23.

24 HYGIN, Fab., 104. 


\section{Le mythe de Phaéthon chez Ovide}

Pour les Grecs, les principaux des personnages que les mythes mettent en scène sont des dieux; ils leur adressent des prières et leur offrent des sacrifices. Hélios, par exemple, fit chez eux l'objet de plusieurs rites. ${ }^{25}$ Certes les mythes évoquent aussi des personnages qui ne reçurent aucun culte mais ces personnages sont le plus souvent proches des dieux; ils ont en outre une telle présence dans l'esprit de chacun que les écrivains peuvent en évoquer pleinement le souvenir en les mentionnant, sans prendre la peine de raconter leur histoire. C'est le cas de Phaéthon auquel la plupart des textes grecs font de simples allusions. La situation est différente à Rome. Bien sûr, Rome a possédé des récits traditionnels originaux; nous en trouvons des exemples remarquables chez TiteLive. Ce ne sont pourtant pas eux qui inspirent habituellement les poètes, ce sont des mythes empruntés à la Grèce ${ }^{26}$. De même Rome a assimilé plusieurs de ses dieux à des divinités helléniques mais, dans la réalité du culte, ses dieux n'en restent pas moins romains; les Lares, les Pénates, Ops, Consus ou Pomone, Faunus, Janus et Mater Matuta n'ont pas d'équivalents grecs et même les grands dieux que l'on rapproche volontiers de figures divines helléniques en diffèrent profondément. Jupiter, Mars et Quirinus, font ensemble l'objet des soins de l'un des flamines majeurs; Jupiter, Minerve et Junon constituent la triade capitoline; or à l'intérieur de ces structures cultuelles, Jupiter est très différent d'un Zeus, Mars, d'un Arès; Junon est très différente d'une Héra et Minerve, d'une Athéna.

Si donc Ovide reprend et raconte des mythes grecs, ceux-ci ne s'inscrivent plus, dans l'esprit de ses lecteurs, à l'intérieur d'un contexte religieux présent dans la mémoire collective ${ }^{27}$. Pourquoi donc Ovide raconte-t-il le mythe de Phaéthon? Quel sens veut-il lui donner? Une première réponse vient immédiatement à l'esprit - et ce n'est pas nécessairement la plus mauvaise - Ovide aime à raconter de belles histoires. Nous ne nous satisferons pourtant pas de cette évidence. Les vers qu'il consacre à Phaéthon constituent seulement un morceau d'une œuvre de grande dimension : elle réunit un nombre élevé de mythes grecs. Où donc Ovide les a-t-il trouvés ? Comment les a-t-il choisis ?

Je dois corriger ce que j'ai dit de la Grèce, il y a un instant. À l'époque hellénistique déjà, la situation n'y est plus celle que j'ai décrite. Lorsque les cités-

25 Éos, Hélios lui-même ne sont pas des divinités majeures dans les cultes grecs. Tels que Zeus, Perséphone ou Dionysos, d'autres personnages mythiques y jouent des rôles beaucoup plus importants. On observera toutefois qu'Hélios, invoqué par des héros homériques (e.g. HOMĖRE, $l l$, III, 104) est l'objet de cultes réguliers en plusieurs lieu de l'Hellade, à Corinthe (PAUSANIAS, II, 4, 6; $11,1 ; 31,5 ; 34,10)$ et à Rhodes ( $c f$. DIODORE DE SICILE V, 56, 3-4), notamment.

26 Observons pourtant qu'OVIDE lui-même évoque certains mythes proprement romains dans les Fastes.

27 La littérature et les arts figurés ont sans doute diffusé une certaine connaissance des traditions helléniques dans le monde romain mais cette connaissance se répand surtout dans les milieux cultivés; elle n'est pas solidaire des traditions religieuses romaines. 
états qui avaient, à l'époque classique, constitué les principales communautés cultuelles de la Grèce ont perdu leur autonomie, les conditions de la vie religieuse se sont transformées et la fonction du mythe a quelque peu changé. Les poètes semblent en jouer parfois, pour le pur plaisir de la narration, et des savants se mettent à les collectionner. C'est ainsi que les lettrés de l'époque alexandrine ont publié des recueils de mythes classés par catégories, en considération des motifs qu'ils mettent en œuvre, aventures amoureuses ou

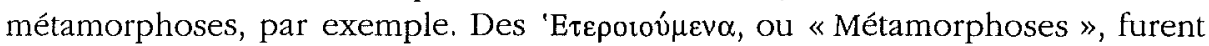
notamment composées par le poète érudit Nicandre, originaire de Colophon, qui vécut sans doute au II $^{\mathrm{e}}$ siècle avant notre ère. Ovide a connu cette œuvre et s'en est probablement inspiré; je ne crois pourtant pas qu'elle soit son unique source.

Je note que le poète de Colophon se plaisait à raconter les aventures de personnages mineurs, découverts dans des traditions locales ${ }^{28}$. Ovide parle aussi de héros mineurs qu'il connaît peut-être grâce à Nicandre mais il traite en outre de mythes centraux dans les traditions helléniques. J'observe une autre chose. L'aventure de Phaéthon n'est précisément pas une métamorphose. Ce sont ses sœurs, les Héliades qui, pleurant la mort de Phaéthon, seront transformées en peupliers; leurs larmes coulant du tronc de l'arbre, sous l'aspect d'une résine, donneront naissance à l'ambre. Cette histoire est associée à celle de Phaéthon, dès l'époque classique. Nous comprenons qu'Ovide évoque la mort de Phaéthon avant de raconter la métamorphose des Héliades mais, en constatant qu'il consacre plus de 350 vers au fils du Soleil, et moins de 35 à ses sœurs, nous reconnaitrons que ce n'est pas la transformation de jeunes filles en arbres qui retient son attention en premier lieu. Il ne paraît donc pas avoir suivi servi lement le modèle d'un répertoire de métamorphoses.

Le cas de Phaéthon n'est pas unique; un bon nombre des histoires racontées par Ovide ne se limitent pas à l'évocation d'une transformation miraculeuse. Le thème de la métamorphose lui permet de coordonner, dans un ensemble relativement homogène, une vaste collection de fables qui lui ont plu. Or tout collectionneur détache chacun des récits choisis du contexte auquel il appartenait primitivement, du système à l'intérieur duquel il remplissait une fonction. Ce faisant, il court le risque de faire perdre au mythe son sens originel; de le réduire à l'état d'un conte surprenant ou pittoresque, effroyable ou touchant.

Ovide a le goût du pittoresque et du merveilleux - songez à la façon dont il décrit le palais, ou le char du Soleil; il donne à ses personnages des sentiments humains, propres à inspirer notre sympathie - songez à l'émotion avec laquelle il dit l'inquiétude du dieu contraint de céder au caprice de son fils; il sait évoquer la catastrophe et suggérer l'épouvante - songez au spectacle qu'il propose de la chute de Phaéthon. Des connaissances érudites nourrissent en outre son imagi-

28 Pour autant qu'il nous soit possible d'en juger en considérant les indications fournies par un compilateur du $\mathrm{II}^{\mathrm{e}}$ ou $\mathrm{III}^{\mathrm{e}}$ siècle de notre ère, Antoninus Liberalis, en considérant d'autre part les quelques fragments conservés du livre de Nicandre. 
nation; il utilise la géographie et l'astronomie pour raconter la course folle du char solaire mais il transforme en monstres fantastiques et menaçants les figures de constellations que les astronomes décrivent. ${ }^{29}$

Négligeant donc la structure du système mythique, Ovide prive le récit ancien de sa portée théologique ou cosmique et tend à lui conférer une signification purement humaine. Une raison plus intéressante le pousse à cela. D'une grande intelligence, supérieur aux autres êtres vivants qu'il est apte à commander, l'homme - lisons-nous dans les Métamorpboses - l'homme apparaît le dernier, dans le cours d'une création qu'il parachève ${ }^{30}$. Or les cosmogonies mythiques de la Grèce nous donnaient un enseignement différent. Ce n'est point l'homme, c'est la perfection du monde qui en constituait l'aboutissement; d'autres êtres vivants, tels que les dieux, le ciel, la terre, les astres, se rangeaient alors au-dessus des hommes. Le sens de la vie et la norme des conduites humaines se trouvaient définis par la situation que les mortels occupent dans l'ordonnance universelle; or elle n'y était pas centrale. Puisque l'homme est donc, aux yeux d'Ovide, supérieurs aux autres êtres, on comprend qu'il concentre sur lui son attention et donne au mythe un contenu psychologique ou moral plutôt qu'un contenu théologique. C'est ainsi qu'il attribue une pratique spontanée de la justice aux hommes du premier âge, alors qu'Hésiode ne dit rien de la qualité morale de la race d'or; de même, il pare de grandes vertus les rescapés du déluge, Deucalion et Pyrrha, ce que les récits grecs ne font pas.

Le mythe grec n'est certes pas dépourvu de toute signification morale mais celle-ci résulte d'une signification première, qui est théo-cosmologique. Si le fils du Soleil naît à l'époque de la séparation entre les mortels et les immortels, lors de crises où les rôles respectifs des uns et des autres se définissent, sa conduite et son échec contribuent à mettre en évidence la situation du mortel dans l'économie religieuse de l'univers et les limites qu'il ne peut franchir impunément. La relation convenable entre les hommes et les dieux ne sera pas une relation

29 Il fait encore autre chose. Il tente de reconstituer un contexte où les mythes groupés arbitrairement puissent trouver une nouvelle raison d'être. S'il raconte une cosmogonie au début de son poème, ce n'est pas simplement pour suggérer l'idée d'une succession chronologique, «de l'origine du monde à notre temps ", comme nous le lisons au début de son premier livre. Il nous montre en effet comment des formes multiples apparaissent dans ce qui est primitivement sans forme et comment les formes se diversifient au cours du temps. Un tel prologue éclaire la suite du récit. Même si elles deviennent mineures et ponctuelles, les transformations ultérieures, constituent une poursuite du processus qui fut en œuvre dans la construction de l'univers. Chaque métamorphose sera une petite création. Celle de Sirynx donne naissance au roseau; celle des Héliades, au peuplier; celle de Cycnus, au cygne; celle de Callisto, à la constellation de l'Ourse. Tous les pays possèdent des contes disant ainsi l'aventure d'un personnage soumis à quelque transformation merveilleuse et qui devient un être nouveau mais, en situant la cosmogonie que nous avons dite au début des Métamorpboses, Ovide confère une densité particulière à ce motif banal. Il ne construit pourtant pas un édifice complet. - Ovide raconte la succession des âges ou le déluge mais il n'y voit plus des événements propres à conférer une structure au temps mythique. Il néglige donc les articulations de ce temps, articulations en considération desquelles il convient de situer chaque récit singulier pour en percevoir le sens.

30 OVIDE, Métam., 1, 76.88. 
amoureuse. Il appartiendra aux dieux, non aux hommes, d'agir sur les événements cosmiques. D'une manière plus générale, Phaéthon est une illustration de ce qui constitue la faute ou la perversité majeure aux yeux des Grecs, cet orgueil et cette démesure qu'ils appellent ößpıs.

Sorti de ce contexte mythique bien structuré propre à la tradition hellénique, Phaéthon perd cette dimension symbolique. Certes elle n'échappe pas complètement à Ovide qui fait dire à Phobus : sors tua mortalis; non est mortale quod optas ${ }^{31}$ mais le poète s'intéresse davantage aux effets spectaculaires de la faute et à la souffrance du fautif qu'à la qualification religieuse des actes qu'il a commis. Son Phaéthon est surtout un malheureux avec lequel nous sympathisons, dont le destin nous terrifie et nous émeut.

Si la figure de Phaéthon reste présente dans la tradition occidentale, c'est grâce au talent d'Ovide; seuls les érudits connaissent les informations que de rares textes grecs survivants nous apportent encore à son sujet. Ainsi, détaché du système mythique auquel il appartenait, ce personnage se prête aujourd'hui à toutes les interprétations imaginables. Gérard de Nerval l'assimile au Christ, tel qu'il le voit, abandonné, désespéré, au Jardin des Oliviers :

C'était bien lui, ce fou, cet insensé sublime...

Cet Icare oublié qui remontait les cieux,

Ce Phaéthon perdu sous la foudre des dieux,

Ce bel Athys meurtri que Cybèle ranime.

Dans son échec, Phaéthon n'est plus ici une illustration de l'ü $\beta \rho \imath c ;$ il devient le symbole d'une aventure spirituelle, noble et grande même si elle paraît insensée.

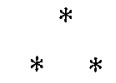

En comparant le mythe de Phaéthon avec le mythe indien signalé par Frazer, nous avions reconnu que les deux récits portent une signification cosmique : bénéfique mais redoutable, le soleil doit être maîtrisé par un être capable de contenir son action dans de justes limites. Nous avions reconnu qu'ils portent aussi une signification psychologique : le fils est enclin à imiter son père mais il ne peut pas s'assimiler complètement à lui; leurs rôles sont différents. Dans son contexte hellénique, le mythe de Phaéthon garde sans doute quelque chose de ces deux significations mais elles s'y précisent en revêtant une portée théologique. Le personnage qui maîtrise le soleil y revêt une identité : c'est un dieu clairement situé dans la société divine, solidaire de l'astre qu'il dirige à tel point que celui-ci peut le signifier aux yeux des Grecs; le dieu est pourtant distinct de lui, toujours au-delà de son apparence solaire. De ce point

31 Ovide, Métam., II, 56. 
de vue l'Hélios du mythe fournit un exemple des relations qui unissent le divin au cosmos; le divin se manifeste dans la vie du cosmos mais il lui demeure transcendant. La relation de filiation devient, elle, le symbole du rapport qui unit le divin à l'humain. L'homme est un descendant des dieux; il participe de leur nature. Le mythe insiste sur la ressemblance qui unit Phaéthon à ses ascendants. Mais la mortalité qui sépare les hommes des dieux leur confère des statuts différents; l'homme doit se connaître; c'est-à-dire connaître ses limites et garder une juste mesure dans toutes ses conduites. Chez Ovide, qui se rattache à la tradition littéraire de la Grèce, le mythe conserve quelque chose de son sens hellénique mais, comme nous l'avons vu, la portée théologique du récit diminue; le poète romain en développe les aspects psychologiques, pour le rendre plus émouvant. Dans la tradition occidentale moderne, le contexte ancien est oublié. Chacun peut utiliser librement la figure de Phaéthon; dans ces conditions, il me paraît intéressant de voir Gérard de Nerval lui attribuer un sens religieux, même s'il diffère du sens théologique que les Grecs lui assignaient. Fou, voué à l'échec, peut-être illégitime, l'envol ou la course vers les cieux suggère l'idée d'une aspiration au divin.

Jean RUDHARDT

1995

17, rue Saint-jean

CH - 1203 GENÈVE 